\title{
Treatment with DAV for Advanced-Stage Hemangiosarcoma in Dogs
}

\author{
Nikolaos G. Dervisis, DVM, DACVIM (Oncology), PhD, Pedro A. Dominguez, DVM, DACVIM (Oncology), \\ DACVR (Radiation Oncology) ${ }^{\star}$, Rebecca G. Newman, DVM, DACVIM (Oncology) ${ }^{\dagger}$, Casey D. Cadile, DVM, \\ DACVIM (Oncology) ${ }^{\S}$, Barbara E. Kitchell, DVM, DACVIM (Oncology, Internal Medicine), PhD
}

\section{ABSTRACT}

\begin{abstract}
Hemangiosarcoma (HSA) is an aggressive disease that is fairly common in the dog. The authors evaluated a doxorubicin, dacarbazine, and vincristine (DAV) combination protocol in dogs with nonresectable stage II and stage III HSA. Twenty-four dogs were enrolled in this prospective, phase 2 study. Doxorubicin and dacarbazine were administered on day 1 while vincristine was administered on days 8 and 15. The protocol was repeated every 21 days for a maximum of six cycles or until disease progression. Toxicity and efficacy were assessed by clinical and laboratory evaluation and by questionnaires completed by the owners. Of the 24 included dogs, 19 were evaluable for response. The response rate (including five complete responses and four partial responses) was $47.4 \%$. Median time to tumor progression was 101 days and median overall survival was 125 days. Significant toxicities were noted, including 41 high-grade hematologic and 12 high-grade gastrointestinal toxic events. Five dogs discontinued treatment due to chemotherapy-related toxicities, but no treatment-related deaths occurred. Multivariate analysis identified patient age (relative risk [RR], 2.3, $P=0.049$ ) to be negatively associated with time to progression whereas dacarbazine dose reductions (RR, $0.06, P=0.031$ ) were positively associated with time to progression. Dacarbazine dose reduction was the sole factor positively associated with overall survival (RR, $0.28, P=0.015)$. In conclusion, the DAV combination appears to offer clinical responses and may prolong survival in dogs with advanced-stage HSA. (J Am Anim Hosp Assoc 2011; 47:170-178. DOI 10.5326/JAAHA-MS-5525)
\end{abstract}

\section{Introduction}

Hemangiosarcoma (HSA) is a malignant tumor that arises from endothelial cells. The disease is fairly common in the dog, representing approximately $5 \%$ of all canine noncutaneous primary malignancies. In contrast, HSA is extremely rare in humans where it represents less than $2 \%$ of all soft tissue sarcomas and only 100 cases of splenic HSA have been reported. ${ }^{1-4}$ German shepherd

From the Center for Comparative Oncology, Michigan State University, East Lansing, MI.

Correspondence: dervisis@cvm.msu.edu (N.D.) dogs, golden retrievers, Labrador retrievers, and schnauzers appear to be predisposed. ${ }^{5,6}$ Canine noncutaneous HSA is typically a highly malignant tumor, frequently metastasizing to distant organs such as the liver, lungs, heart, skin, and central nervous system. ${ }^{7,8}$ Many patients present with internal bleeding due to tumor rupture or disseminated intravascular coagulation., ${ }^{2,9-12}$ Surgery may be palliative to arrest active hemorrhage, but does
CBC complete blood count; CR complete response; DAV doxorubicin, dacarbazine, and vincristine; DFI disease-free interval; DTIC dacarbazine; HSA hemangiosarcoma; IV intravenously; MTD maximum tolerated dose; OS overall survival; PD progressive disease; PR partial response; RR relative risk; SD stable disease; TTP time to progression

${ }^{*}$ P. Dominguez's present address is Animal Cancer Care Clinic, Deerfield Beach, FL.

${ }^{\dagger} \mathrm{R}$. Newman's present address is Pittsburgh Veterinary Specialty and Emergency Center, Pittsburgh, PA.

${ }^{\S} \mathrm{C}$. Cadile's present address is Veterinary Medicine Specialists, San Mateo, CA. 
not provide long-term survival for the majority of cases with visceral involvement. ${ }^{8,11,13,14}$

Chemotherapy in the adjuvant setting has been evaluated in various clinical studies and has shown promise in prolonging life after surgical resection of the primary HSA lesion. ${ }^{15-19}$ Most of those studies included dogs with different clinical stages of disease. It appears that dogs with gross metastatic disease at the time of diagnosis (i.e., stage III) carried significantly worse prognoses, with reported median survival durations between 68 and 136 days. ${ }^{17,20,21}$

The most effective chemotherapy protocols for the treatment of canine HSA are anthracycline-based. Doxorubicin and its analog epirubicin have been used: as single agents in the standard $3 \mathrm{wk}$ protocol; in a dose-intense fashion (2 wk protocol); in combination with cyclophosphamide; or with cyclophosphamide and vincristine. $^{20-26}$ There have been no studies specifically evaluating the treatment of dogs with grossly metastatic HSA, a common presentation in the cases of HSA. ${ }^{8,10,12}$ Nonresectable, primary, noncutaneous HSA appears to carry a negative prognosis for overall survival (OS), yet the use of chemotherapy or palliative radiation therapy has been largely unexplored. ${ }^{27}$

This study evaluated the efficacy of a combination chemotherapy protocol comprised of doxorubicin, dacarbazine, and vincristine (DAV) for the treatment of advanced-stage canine noncutaneous HSA. Dogs were considered to have advanced-stage disease when a nonresectable, primary tumor and/or gross metastatic disease were present at the time of diagnosis. Dacarbazine (DTIC) is an alkylating agent used in the treatment of relapsed round cell tumors, high-grade sarcomas, and malignant melanomas. ${ }^{28-31}$ Results of both in vitro and in vivo studies suggest that DTIC acts synergistically with anthracyclines and has a moderate effect in the treatment of high-grade sarcomas in humans. ${ }^{32-36}$ Vincristine has demonstrated modest responses in a limited number of canine HSA patients. ${ }^{25,37}$ The authors hypothesized that the DAV combination would be effective in providing clinical responses in dogs with advanced-stage noncutaneous HSA.

\section{Materials and Methods}

\section{Animals}

Dogs examined at the Center for Comparative Oncology at Michigan State University between Jan 2004 and Dec 2006 with newly diagnosed HSA were eligible for inclusion in the study. The study was designed and performed in compliance with the Michigan State University Institutional Animal Care and Use Committee guidelines for research on animals and owner informed consent was obtained. Dogs diagnosed with HSA were included only if they had not received any type of chemotherapy treatment. In addition, dogs were included only if the diagnosis had been confirmed on the basis of results of histologic or cytologic examination. For the cytologic examination to be consistent with the diagnosis of HSA, all the following criteria had to be satisfied: cytologic diagnosis of sarcoma; cytologic diagnosis of pathologic hemorrhage; and confirmation of a fluid-filled multicavitated tumor lesion via ultrasonographic examination. ${ }^{38-40}$ Dogs were considered to have a high-risk of developing doxorubicin-related cardiotoxicosis if systolic fractional shortening determined by echocardiography was $<25 \%$. These dogs were excluded from the study. Dogs with life-limiting, non-neoplastic comorbid conditions were also excluded from the study.

\section{Treatment Protocol}

Doxorubicin $^{\mathrm{a}}$ and $\mathrm{DTIC}^{\mathrm{b}}$ were administered on day 1 while vincristine $^{c}$ was administered on days 8 and 15 . The protocol was repeated every 21 days for a maximum of six cycles or until disease progression. Doxorubicin was administered through an indwelling intravenous (IV) catheter over $20 \mathrm{~min}$ at a dose of $30 \mathrm{mg} / \mathrm{m}^{2}$ (diluted in $50-100 \mathrm{~mL}$ of $0.9 \% \mathrm{NaCl}$ ). DTIC was administered immediately after doxorubicin administration through the same catheter at a dose of $800 \mathrm{mg} / \mathrm{m}^{2}$ over $8 \mathrm{hr}$ (diluted in $0.9 \% \mathrm{NaCl}$ ). Dogs were pretreated with $4 \mathrm{mg}$ dexamethasone sodium phosphate ${ }^{\mathrm{d}}$ IV and butorphanol ${ }^{\mathrm{e}}(0.4 \mathrm{mg} / \mathrm{kg}$ intramuscularly) before each DTIC treatment. Metoclopramide ${ }^{\mathrm{f}}(0.5 \mathrm{mg} / \mathrm{kg})$ was dispensed to the owners to be administered orally as needed at home. Vincristine was administered through a butterfly catheter IV as a bolus at a dose of $0.5 \mathrm{mg} / \mathrm{m}^{2}$.

Dose reductions were allowed only for DTIC in the case of grade 3 or 4 toxicity, in $100 \mathrm{mg} / \mathrm{m}^{2}$ increments. No dose escalations were allowed for any of the three chemotherapy drugs.

\section{Evaluation of Response and Toxicity}

In all dogs, a complete blood count (CBC) and complete physical examination were performed on days $1,7,15$, and 22 of treatment. An echocardiographic evaluation of cardiac function was performed before the first doxorubicin and DTIC administration. An electrocardiogram was performed before each subsequent treatment with doxorubicin and DTIC to monitor for changes associated with doxorubicin-induced cardiotoxicity. ${ }^{41,42}$ Thoracic radiography and abdominal ultrasonography were performed every two treatment cycles (i.e., every $6 \mathrm{wk}$ ) for the duration of the chemotherapy protocol and every 3 mo afterward to define response to treatment. Evidence of progressive primary disease and new ultrasonographic lesions were investigated by means of cytologic examination of fine-needle aspirates. Lesions appearing cavitated under ultrasonographic examination were considered to be positive for HSA even when cytology was negative for neoplasia. Dogs were 
considered lost to follow-up when not returned for scheduled examinations and the veterinarian or owner could not be contacted despite repeated attempts. Dogs lost to follow-up were censored.

Tumor response was determined each time the dogs were examined. A complete response (CR) was defined as disappearance of all measurable disease for at least 21 days. A partial response (PR) was defined as a $>50 \%$ but $<100 \%$ reduction in measurable disease for at least 21 days. Stable disease (SD) was defined as a $<50 \%$ reduction in measurable disease for at least 21 days with no appearance of new lesions during that period. Progressive disease (PD) was defined as a $>25 \%$ increase in measurable disease or appearance of new lesions. Transient decreases in measurable disease that persisted for $<21$ days and were followed by increased tumor size were also defined as progressive disease.

Toxicoses were identified on the basis of history and results of physical examinations and CBCs. Criteria established by the Veterinary Co-operative Oncology Group were used to grade toxicoses (Table 1). ${ }^{43}$

\section{Statistical Analysis}

The study was structured with a two-stage design to allow early study closure if the overall clinical benefit rate was unacceptably low. ${ }^{44,45}$ In the first stage, 10 patients were entered. If two or more clinical responses were seen in the first stage of the study, then an additional 12 patients were entered into the study for a planned total of 22 cases. If seven or more clinical responses were observed in total, then the conclusion was that the chemotherapeutic protocol was effective. If less than seven responses were noted in the total patient sample population, then the conclusion was that there was insufficient anticancer activity of the DAV protocol to support continued evaluation. This study was designed for a Type I error of 0.05 and a Type II error of 0.1 with sufficient power to distinguish a clinically promising response rate of 0.5 from an unpromising response rate of 0.2 .

Time to progression (TTP) was defined as the time from initiation of the chemotherapy treatment until disease progression. Disease-free interval (DFI) was defined as the time from the initiation of the chemotherapy treatment until disease relapse for those dogs that achieved complete remission. OS was defined as the time from initiation of the chemotherapy protocol until patient death. The Kaplan-Meier survival analysis method was used to estimate clinical responses and survival time curves following treatment. The Log-Rank test was used to compare the effect of potential risk factors (i.e., gender, age, body weight, stage of disease, median dose of DTIC, DTIC dose reductions, splenic primary site, lung metastasis on presentation, peripheral blood hematocrit before treatment, and peripheral blood platelet number

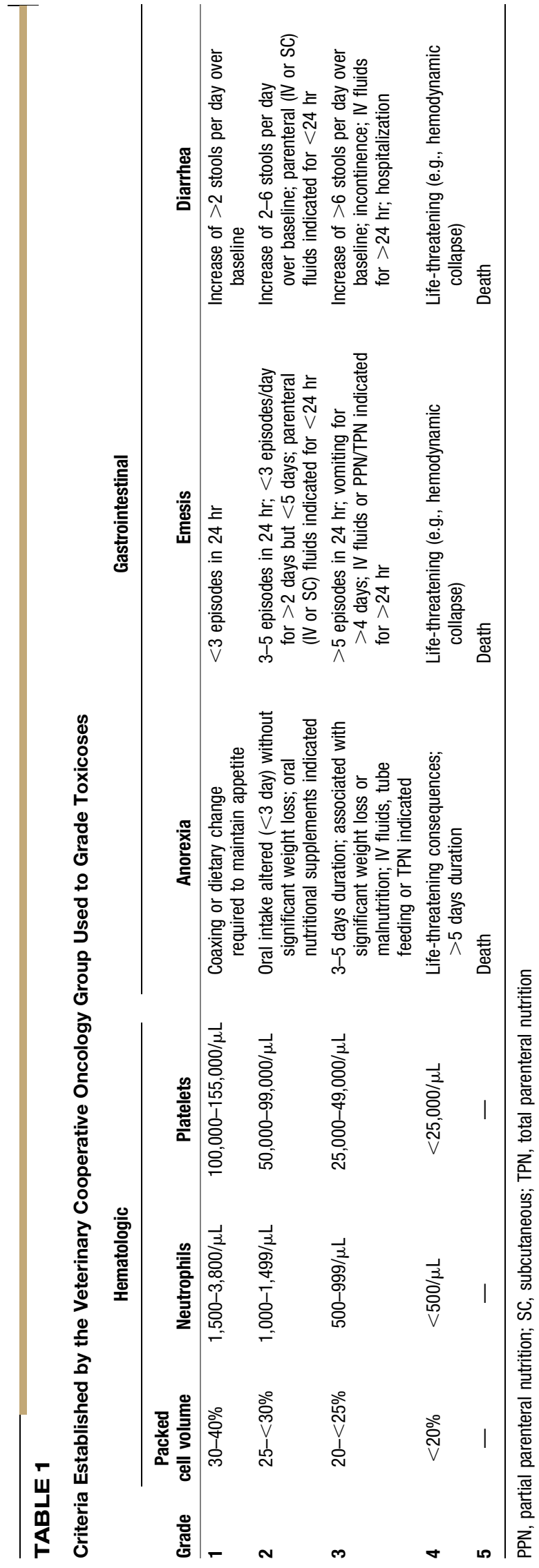


before treatment) to DFI, TTP, and OS. The Cox proportional hazards regression method was used to determine whether potential risk factors (i.e., gender, age, body weight, stage of disease, median dose of DTIC, DTIC dose reductions, splenic primary site, lung metastasis on presentation, peripheral blood hematocrit before treatment, and peripheral blood platelet number before treatment) were associated with TTP or OS following chemotherapy. The potential risk factors were entered in the regression model if their $P<0.05$ and removed if $P>0.1$. All reported $P$ values were 2 -sided and $P$ values $<0.05$ were considered significant. Statistical analyses were performed with standard software ${ }^{\mathrm{g}}$.

\section{Results}

\section{Patients}

Twenty-four dogs were enrolled in the study, including 14 males. Breeds represented in the study were: German shepherds $(n=7)$, golden retrievers $(n=6)$, Labrador retrievers $(n=2)$, beagle $(n=1)$, American pitt bull terrier $(n=1)$, English setter $(n=1)$, and mixed-breed $(n=6)$. The mean age of all dogs was $10.4 \mathrm{yr} \pm 1.8 \mathrm{yr}$ (standard deviation) and mean body weight was $32.4 \mathrm{~kg} \pm 8.4 \mathrm{~kg}$. Twenty dogs had evidence of metastatic HSA at presentation and four dogs had large, nonresectable primary HSA. Of the 20 dogs with stage III HSA, 15 had surgical resection of the primary tumor and attempted resection of metastases. Successful resection of all gross primary and metastatic disease was achieved in three dogs. Five dogs with metastatic disease were characterized as poor surgical candidates and therefore did not undergo surgery before inclusion in this study. None of the four dogs with stage II disease underwent surgical resection of the primary tumor due to tumor size and location. The stage, primary tumor site, and whether surgery was performed before chemotherapy have been summarized in Table 2. Metastatic sites at the time of presentation included liver $(n=9,37.5 \%)$, lungs $(n=7,29.5 \%)$, omentum $(n=2$, $8 \%)$, retroperitoneum $(n=2,8 \%)$, spleen $(n=1,4.25 \%)$, subcutaneous tissues $(n=1,4.25 \%)$, bone $(n=1,4.25 \%)$, and mesentery $(n=1,4.25 \%)$.

A median of 3.5 cycles (range, 1-6 cycles) of DAV was administered with 79 cycles administered in total. The median cumulative dose of doxorubicin was $90 \mathrm{mg} / \mathrm{m}^{2}$ (range, 30-180 $\mathrm{mg} / \mathrm{m}^{2}$ ). The median DTIC administered dose was $785.5 \mathrm{mg} / \mathrm{m}^{2}$ (range, $500-800 \mathrm{mg} / \mathrm{m}^{2}$ ) and the median cumulative dose was $2,600 \mathrm{mg} / \mathrm{m}^{2}$ (range, $500-4,600 \mathrm{mg} / \mathrm{m}^{2}$ ). The median cumulative dose of vincristine was $3.5 \mathrm{mg} / \mathrm{m}^{2}$ (range, $0.5-6 \mathrm{mg} / \mathrm{m}^{2}$ ).

\section{Efficacy}

Only 19 of the 24 dogs were evaluable for response because three dogs had no evidence of disease after surgery and were therefore

\section{TABLE 2}

Stage, Primary Tumor Site, and Surgery Prior to Starting Chemotherapy with a Combination of Doxorubicin, Dacarbazine, and Vincristine (DAV) in Dogs $(n=24)$

\begin{tabular}{lllcc} 
& & Primary site & $\begin{array}{c}\text { Number of dogs } \\
(\mathbf{n}=24)\end{array}$ & $\begin{array}{c}\text { Surgery before } \\
\text { chemotherapy? }\end{array}$ \\
\hline Stage & II & Subcutaneous & 2 & no \\
& & Bone & 1 & no \\
Stage & Heart & SII & 1 & no \\
& & Spleen & 12 & yes $(\mathrm{n}=12)$ \\
& Heart & 2 & yes $(\mathrm{n}=1)$ \\
& Subcutaneous & 1 & no \\
& & Bone & 1 & yes $(\mathrm{n}=1)$ \\
& Liver & 1 & no \\
& Lungs & 1 & no \\
& Kidney & 1 & yes $(\mathrm{n}=1)$ \\
& Vagina & 1 & no \\
\hline
\end{tabular}

treated in an adjuvant setting and two dogs were lost to follow-up. A CR occurred in 5 of the 19 dogs (26.3\%), 4/19 (21.1\%) dogs had a PR, 9/19 dogs (47.4\%) had SD, and 1/19 (5.3\%) had PD during the course of DAV treatment.

The median DFI for dogs that achieved CR was 205 days (range, 82-400 days). The median TTP for dogs that either had a clinical response (CR or PR) or SD was 101 days (range, 21-400 days, Figure 1). Median OS for all 24 dogs in the study was 125 days (range, 18-411 days, Figure 2).

Twenty-two dogs were euthanized due to HSA progression. Of the two dogs not euthanized due to PD, one dog was euthanized due to acute renal failure while in CR and the other dog was found dead by the owners 1 day after a complete restaging (i.e., CBC, serum chemistry, urinalysis, thoracic radiographs, abdominal

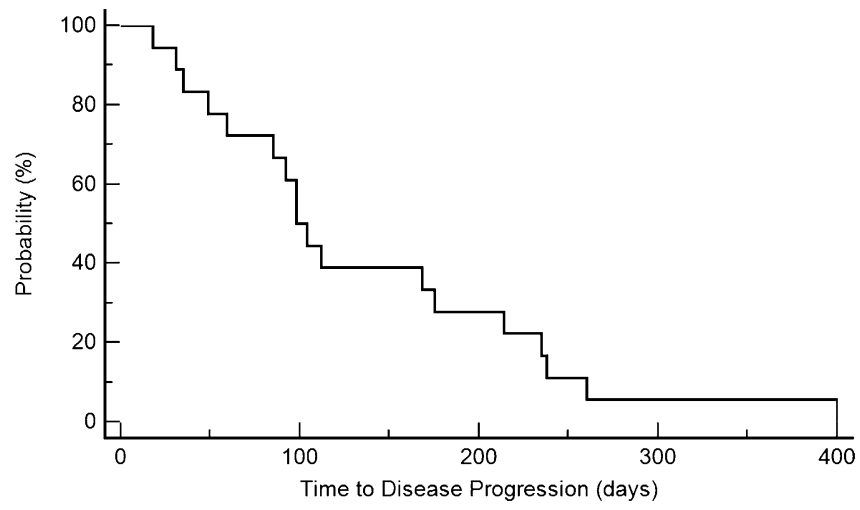

FIGURE 1 Time to progression (TTP) of patients $(n=18)$ with advanced-stage hemangiosarcoma (HSA) achieving a complete response $(C R)$, partial response $(P R)$, or stable disease $(S D)$ following treatment with a combination of doxorubicin, dacarbazine, and vincristine (DAV). 


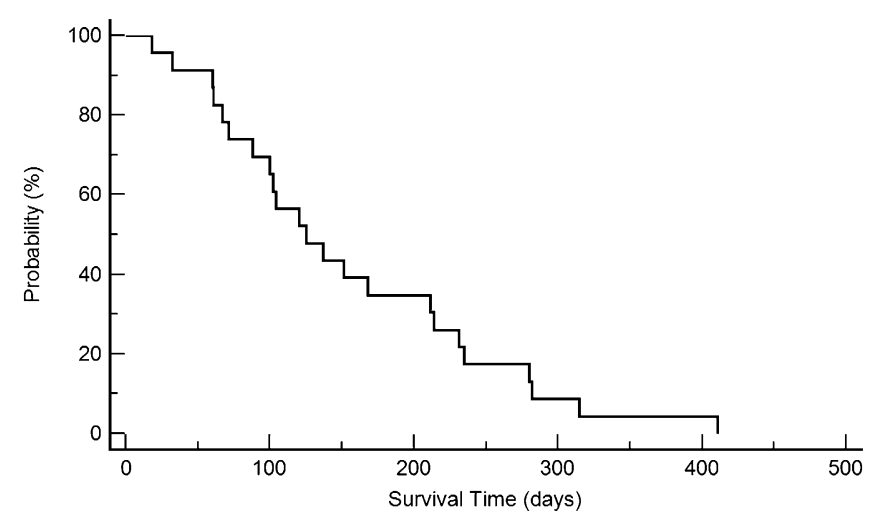

FIGURE 2 Overall survival of patients with advanced HSA treated with DAV $(n=24)$.

ultrasound) demonstrated a CR. The owners of both of these latter two dogs declined necropsy. Two of four dogs with advanced stage II disease were euthanized due to local disease progression 214 and 282 days after starting DAV. The other two dogs with advanced stage II disease developed metastatic disease and were euthanized 71 and 125 days after starting DAV.

\section{Toxicity}

The most common treatment-related toxicoses were hematologic and gastrointestinal. Overall, 221 hematologic toxic events were noted in the 24 dogs. Of these toxicities, 23 were grade 3 and 18 were grade 4 . Grade 3 toxicities included anemia $(n=9)$, neutropenia $(n=12)$, and thrombocytopenia $(n=2)$. Grade 4 toxicities included anemia $(n=4)$, neutropenia $(n=10)$, and thrombocytopenia $(n=4)$. A total of 96 events of gastrointestinal toxicoses were observed. Of these events, eight were grade 3 , and four were grade 4 toxicities. Grade 3 toxicities included anorexia $(n=1)$, emesis $(n=3)$, and diarrhea $(n=4)$. Grade 4 toxicities included emesis $(n=2)$ and diarrhea $(n=2)$.

The mean hematocrit of the treated dogs at the beginning of their therapy with the DAV protocol was $36.1 \%$ ( $\pm 5.16 \%$ ). At the end of the treatment protocol, the mean hematocrit was $33.6 \%$ ( $\pm 7.8 \%$; reference range, $40-55 \%$ ). The mean platelet number at the beginning of the treatment with the DAV protocol was $382 \times$ $\left.10^{3} / \mu \mathrm{L} \pm 237 \times 10^{3} / \mu \mathrm{L}\right)$. At the end of the treatment protocol, the mean platelet number was $451 \times 10^{3} / \mu \mathrm{L} \pm 179 \times 10^{3} / \mu \mathrm{L}$ (reference range, $155-393 \times 10^{3} / \mu \mathrm{L}$ ). These differences were not statistically significant.

Five out of 24 dogs were hospitalized due to side effects of the chemotherapy for a median of 4 days (range, 2-9 days). The hospitalized dogs had concurrent hematologic and gastrointestinal high-grade toxicoses. All dogs manifested the severe toxicoses after administration of the doxorubicin and DTIC. No dogs died or were euthanized due to treatment-related toxicoses, but four of these five dogs discontinued chemotherapy because of the adverse effects. Additionally, one dog discontinued treatment due to toxicity (as assessed by its owner) despite the fact that this dog did not require hospitalization.

\section{Risk Factor Analysis}

Univariate analysis of risk factors indicated that dogs with nonsplenic primary HSA had a favorable median DFI of 307 days versus dogs with splenic primary HSA that achieved a median DFI of 104 days ( $P=0.0136$ ). The median dose of DTIC administered was positively associated with a longer TTP $(P=0.0041)$. Dogs that had to be treated with a reduced dose of DTIC due to toxicity $(n=11)$ had a longer median OS (211 days) compared with dogs that did not require dose reductions (median $\mathrm{OS}=100$ days, $P=0.0159)$. The median dose of DTIC was positively associated with a longer OS $(P=0.004)$. Dogs that stopped chemotherapy treatment early due to toxicity $(n=4)$ had a median OS of 79.5 days compared with dogs that tolerated the protocol and achieved a median OS of 151 days $(P=0.0252)$. Dogs that had metastatic disease to the lung parenchyma at the time of diagnosis $(n=7)$ had a median OS of 67 days compared with dogs presenting with metastatic disease outside of the lung parenchyma $(n=13)$ that achieved a median OS of 151 days $(P=0.0365)$.

Multivariate analysis identified age (relative risk [RR], 2.3, $P=0.049$ ) to be negatively associated with TTP (Figure 3). DTIC dose reductions ( $\mathrm{RR}, 0.06, P=0.031$ ) were positively associated with TTP (Figure 4). DTIC dose reduction was the sole factor positively associated with $\mathrm{OS}(\mathrm{RR}, 0.28, P=0.015)$.

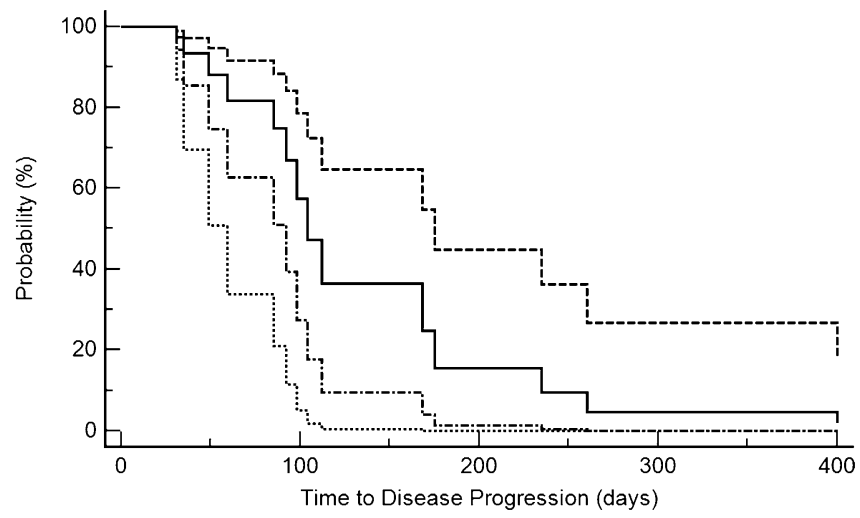

FIGURE 3 Age as a risk factor $(R R, 2.3, \mathrm{P}=0.049)$ for disease progression in dogs with advanced HSA treated with DAV. The dotted line represents the 75-100\% age quartile (11.5-14.2 yr), the dashed-dotted line represents the 50-75\% age quartile (10.3-11.5 yr), the solid line represents the 25-50\% age quartile (9.7-10.3 $\mathrm{yr}$ ), and the dashed line represents the 0-25\% age quartile (5.5-9.7 yr). 


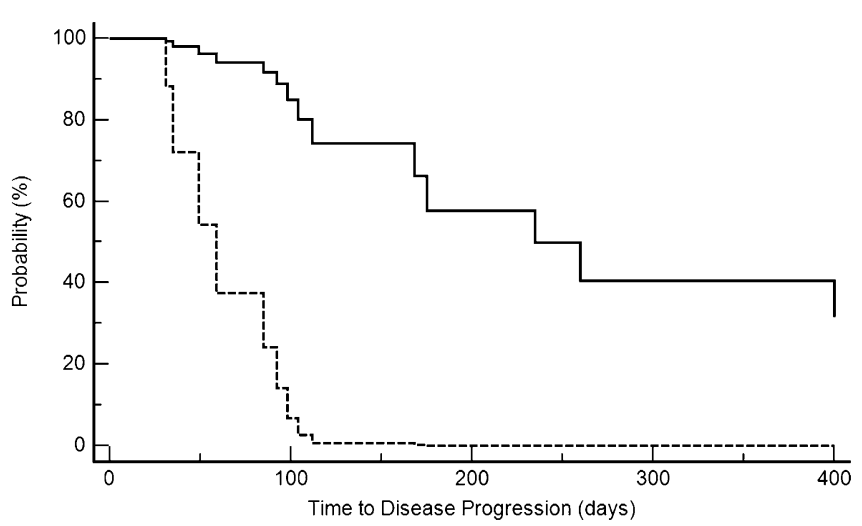

FIGURE 4 Dacarbazine (DTIC) dose reductions due to toxicity reduced the risk of disease progression $(R R, 0.06, \mathrm{P}=0.031)$. The solid line represents patients having DTIC dose reductions due to toxicity and the dashed line represents patients without DTIC dose reductions.

\section{Additional Treatments}

Six dogs received ifosfamide ${ }^{\mathrm{h}}$ rescue treatment at tumor progression and 18 dogs did not receive any rescue chemotherapy treatment. No response was seen in the ifosfamide treated dogs. Two dogs with advanced stage II disease received palliative radiation therapy (three weekly $8 \mathrm{~Gy}$ fractions using $6 \mathrm{MeV}$ photons), but demonstrated no clinical improvement of local disease. One dog finished six cycles of the DAV protocol in CR and continued to be treated with a combination of dactinomycin ${ }^{i}$ and temozolomide ${ }^{j}$ once a month until tumor relapse for a total of 12 treatments. Four dogs finished the DAV protocol in CR and were included in a phase 1 study of a metronomic chemotherapeutic protocol.

\section{Discussion}

Dogs with noncutaneous HSA are generally considered to have a poor prognosis. Only a relatively small percentage of dogs achieve long-term survival. ${ }^{16,17,20,21,23-26}$ Dogs presenting with advancedstage disease are considered to have a grave prognosis and, many times, are not offered any treatment options other than hospice or euthanasia. In the study described herein, the authors demonstrate the activity of a combination chemotherapy protocol that is based on administration of doxorubicin and DTIC on the same day, combined with vincristine administration. These results indicate that even dogs with either widespread, metastatic or nonresectable, noncutaneous HSA can benefit from aggressive doxorubicin-based chemotherapy, achieving a CR of $26.3 \%$ and a PR of $21.1 \%$ (for a total response rate of $47.4 \%$ ). Despite the overall poor prognosis in this disease setting, dogs achieving a CR had a median DFI of 205 days.
The patients with the longest survival were those that achieved CR either through chemotherapy alone or being treated in an adjuvant setting after complete surgical removal of all gross primary and metastatic disease. The difference in survival between dogs that were treated in an adjuvant setting and those treated in the gross disease setting was not statistically significant, presumably due to the small number of dogs in the adjuvant treatment group $(n=3)$. The survivals for those three dogs were 231, 235, and 411 days. Formal power analysis in this patient sample set indicated that, with an $\alpha$ of 0.05 and power of $80 \%, 20$ dogs were required for each of the comparator groups (i.e., dogs treated with aggressive surgical resection of all primary and metastatic disease versus dogs treated with conservative surgical resection of only the bleeding tumors and followed by chemotherapy). Thus, a larger study would be required to assess the validity of this last observation.

One interesting and unexpected finding in this study was the positive association of DTIC dose reductions with increased TTP and OS. In theory, lower doses of chemotherapy are associated with decreased cancer cell killing. The authors believe that these study results reflect the need to treat advanced-stage, noncutaneous HSA with the maximum tolerated dose (MTD) of DTIC. DTIC is a prodrug that requires enzymatic activation via members of the cytochrome-P450 system (CYP1A1, CYP1A2, and CYP2E1) to exert its cytotoxic effect. ${ }^{46}$ There is evidence indicating that genetic polymorphisms in these CYP genes can have profound effects on the pharmacokinetics of CYP-dependent drugs. ${ }^{47-52}$ It is therefore possible that some dogs tolerate higher doses of DTIC than others due to slower activation of the drug and a subsequent smaller area under the drug concentration-time curve. Due to the study design, the DTIC dose was not escalated so it was not possible to assess the individual patients' MTD if they tolerated the standard dose of $800 \mathrm{mg} / \mathrm{m}^{2}$. On the other hand, animals that demonstrated significant toxicity were subsequently treated with lower doses of DTIC, achieving a treatment closer to their individual MTD, which reduced the apparent risk of disease progression. Pharmacogenomic and pharmacokinetic studies of DTIC and its metabolites triazine 3-methyl-(triazen-1-yl) imidazole-4-carboxamide (MTIC) and 5-amino-imidazole-4carboxamide (AIC) in the canine cancer patient would be invaluable in deriving future dosing schema.

The toxicity of the DAV protocol appears to be significant. Five dogs were withdrawn from the study by their owners due to hematologic and/or gastrointestinal toxicities. All toxic events were observed after the administration of doxorubicin and DTIC. None of these five dogs received any type of additional chemotherapy after the toxic events. Further, the median OS of these five dogs was significantly shorter than the remaining patients included in the 
study. Two of these five dogs were lost to follow-up and no data regarding their tumor responses were available. Two dogs achieved a PR with only one treatment of doxorubicin and DTIC and one dog had SD. One of the dogs achieving a PR had nonresectable, primary, right atrial HSA ( $O S=241$ days). The second dog had retroperitoneal HSA with widespread lung metastases (OS=73 days).

No evidence of clinical cardiotoxicity was noted in this study. This finding could be attributed to the relatively stringent entry criteria with regard to cardiac function, the limited number of dogs in the study, the low median number of doxorubicin treatments administered (median=3.5 treatments), and the relative low median survival of the dogs in this study.

Efforts to alleviate the gastrointestinal and hematologic toxicities could be made in future studies. Newer antiemetic drugs are currently available that were not commercially available during the study period, such as maropitant ${ }^{\mathrm{k}}$. Use of improved antiemetic strategies could help to better control chemotherapy-related emesis and reduce the dehydration risk. The neutropenic sepsis risk could be reduced using prophylactic antibiotics or growth factors, such as human recombinant granulocyte colony stimulating factor, after the doxorubicin and DTIC administration. Furthermore, DTIC dose optimization could result in a reduction of treatment-related toxicity. This study was designed as a single arm, phase 2 study. The primary endpoint was the response rate and secondary endpoints were TTP, DFI, and frequency and severity of treatment-related toxicities. The study design required a minimum of 22 dogs in total, but 2 additional dogs that presented to the clinic were also included to increase the power of the study. The OS data should be evaluated with care since six of the dogs that had PD or that relapsed during treatment with the DAV protocol received rescue treatment with ifosfamide. Four dogs that finished the DAV protocol in CR were assigned to a metronomic protocol as part of an independent phase 1 study. Additionally, the OS was defined from the start of the DAV protocol and not from the time of surgery or the time of diagnosis. Thus, even though no dog responded favorably to rescue treatments and the effect of our metronomic treatment was difficult to assess at the time this report was written, the OS data should be interpreted under the aforementioned conditions.

This study had a number of limitations. The authors attempted to have a homogeneous sample of animals with noncutaneous HSA carrying the worst prognosis. It is well understood by the authors that including dogs with advanced stage II disease might have increased the biologic variability in the study. Additionally, a small number $(n=3)$ of dogs in the study had only a cytologic diagnosis of HSA. These three dogs had stage III disease with tumors that were difficult to obtain tissue samples for histopathologic diagnosis. Therefore, the authors relied on cytologic diagnosis with strong clinical and imaging criteria that pointed toward HSA. It is possible that some of these tumors were other types of high-grade, metastatic sarcomas, but these dogs' responses to treatment, TTPs, and OSs were not significantly different to dogs for which the diagnosis was based on histopathology. Therefore, these three dogs were included in the overall analysis.

\section{Conclusion}

The DAV protocol appears to be active against advanced-stage, noncutaneous HSA in the dog. Further phase 3 clinical studies are needed to compare the efficacy and toxicity of different doxorubicin-based protocols in a large population of dogs with distinct clinical stages.

\section{FOOTNOTES}

a Adriamycin; Bedford Laboratories Inc., Bedford, $\mathrm{OH}$

b Dacarbazine; American Pharmaceutical Partners Inc., Schaumburg, IL

c Vincristine sulfate; Mayne Pharma, Paramus, NJ

d Dexamethazone; American Reagent, Inc., Shirley, NY

e Butorphanol; Intervet INC., Milsboro, DE

f Metoclopramide; Baxter Healthcare Corp., Deerfield, IL

g MedCalc for Windows, version 10.0.0.0; MedCalc Software, Mariakerke, Belgium

h Ifosfamide; Bristol Myers Squibb Company, Princeton, NJ

Dactinomycin; Ovation Pharmaceuticals, Deerfield, IL

Temozolomide; Shering Corp., Kenilworth, NJ

k Maropitant, Cerenia, Pfizer Animal Health, New York, NY

\section{REFERENCES}

1. Priester WA. Hepatic angiosarcomas in dogs: an excessive frequency as compared with man. J Natl Cancer Inst 1976;57(2): $451-4$.

2. Prymak C, McKee LJ, Goldschmidt MH, et al. Epidemiologic, clinical, pathologic, and prognostic characteristics of splenic hemangiosarcoma and splenic hematoma in dogs: 217 cases (1985). J Am Vet Med Assoc 1988;193(6):706-12.

3. Rupolo M, Berretta M, Buonadonna A, et al. Metastatic angiosarcoma of the spleen. A case report and treatment approach. Tumori 2001;87(6):439-43.

4. Hsu JT, Chen HM, Lin CY, et al. Primary angiosarcoma of the spleen. J Surg Oncol 2005;92(4):312-6.

5. Clifford CA, Mackin AJ, Henry CJ. Treatment of canine hemangiosarcoma: 2000 and beyond. J Vet Intern Med 2000;14(5): 479-85.

6. Brown NO. Hemangiosarcomas. Vet Clin North Am Small Anim Pract 1985;15(3):569-75.

7. Snyder JM, Lipitz L, Skorupski KA, et al. Secondary intracranial neoplasia in the dog: 177 cases (1986-2003). J Vet Intern Med 2008; 22(1):172-7. 
8. Brown NO, Patnaik AK, MacEwen EG. Canine hemangiosarcoma: retrospective analysis of 104 cases. J Am Vet Med Assoc 1985;186(1): $56-8$.

9. Legendre AM, Krehbiel JD. Disseminated intravascular coagulation in a dog with hemothorax and hemangiosarcoma. J Am Vet Med Assoc 1977;171(10):1070-1.

10. Ng CY, Mills JN. Clinical and haematological features of haemangiosarcoma in dogs. Aust Vet J 1985;62(1):1-4.

11. Johnson KA, Powers BE, Withrow SJ, et al. Splenomegaly in dogs. Predictors of neoplasia and survival after splenectomy. J Vet Intern Med 1989;3(3):160-6.

12. Hammond TN, Pesillo-Crosby SA. Prevalence of hemangiosarcoma in anemic dogs with a splenic mass and hemoperitoneum requiring a transfusion: 71 cases (2003-2005). J Am Vet Med Assoc 2008; 232(4):553-8.

13. Wood CA, Moore AS, Gliatto JM, et al. Prognosis for dogs with stage I or II splenic hemangiosarcoma treated by splenectomy alone: 32 cases (1991-1993). J Am Anim Hosp Assoc 1998;34(5):417-21.

14. Kerstetter KK, Krahwinkel DJ Jr, Millis DL, et al. Pericardiectomy in dogs: 22 cases (1978-1994). J Am Vet Med Assoc 1997;211(6): 736-40.

15. U'Ren LW, Biller BJ, Elmslie RE, et al. Evaluation of a novel tumor vaccine in dogs with hemangiosarcoma. J Vet Intern Med 2007; 21(1):113-20.

16. Lana S, U'ren L, Plaza S, et al. Continuous low-dose oral chemotherapy for adjuvant therapy of splenic hemangiosarcoma in dogs. $J$ Vet Intern Med 2007;21(4):764-9.

17. Kim SE, Liptak JM, Gall TT, et al. Epirubicin in the adjuvant treatment of splenic hemangiosarcoma in dogs: 59 cases (1997-2004). J Am Vet Med Assoc 2007;231(10):1550-7.

18. Vail DM, MacEwen EG, Kurzman ID, et al. Liposome-encapsulated muramyl tripeptide phosphatidylethanolamine adjuvant immunotherapy for splenic hemangiosarcoma in the dog: a randomized multi-institutional clinical trial. Clin Cancer Res 1995;1(10): $1165-70$.

19. MacEwen EG, Kurzman ID, Helfand S, et al. Current studies of liposome muramyl tripeptide (CGP 19835A lipid) therapy for metastasis in spontaneous tumors: a progress review. J Drug Target 1994;2(5):391-6.

20. Sorenmo KU, Baez JL, Clifford CA, et al. Efficacy and toxicity of a dose-intensified doxorubicin protocol in canine hemangiosarcoma. J Vet Intern Med 2004;18(2):209-13.

21. Sorenmo K, Duda L, Barber L, et al. Canine hemangiosarcoma treated with standard chemotherapy and minocycline. J Vet Intern Med 2000;14(4):395-8.

22. Sorenmo K, Samluk M, Clifford C, et al. Clinical and pharmacokinetic characteristics of intracavitary administration of pegylated liposomal encapsulated doxorubicin in dogs with splenic hemangiosarcoma. J Vet Intern Med 2007;21(6):1347-54.

23. Ogilvie GK, Powers BE, Mallinckrodt CH, et al. Surgery and doxorubicin in dogs with hemangiosarcoma. J Vet Intern Med 1996; 10(6):379-84.

24. Sorenmo KU, Jeglum KA, Helfand SC. Chemotherapy of canine hemangiosarcoma with doxorubicin and cyclophosphamide. J Vet Intern Med 1993;7(6):370-6.

25. Hammer AS, Couto CG, Filppi J, et al. Efficacy and toxicity of VAC chemotherapy (vincristine, doxorubicin, and cyclophosphamide) in dogs with hemangiosarcoma. J Vet Intern Med 1991;5(3): $160-6$.
26. Ogilvie GK, Reynolds HA, Richardson RC, et al. Phase II evaluation of doxorubicin for treatment of various canine neoplasms. J Am Vet Med Assoc 1989;195(11):1580-3.

27. Aronsohn M. Cardiac hemangiosarcoma in the dog: a review of 38 cases. J Am Vet Med Assoc 1985;187(9):922-6.

28. Ahaus EA, Couto CG, Valerius KD. Hematological toxicity of doxorubicin-containing protocols in dogs with spontaneously occurring malignant tumors. J Am Anim Hosp Assoc 2000;36(5):422-6.

29. Dervisis NG, Dominguez PA, Sarbu L, et al. Efficacy of temozolomide or dacarbazine in combination with an anthracycline for rescue chemotherapy in dogs with lymphoma. J Am Vet Med Assoc 2007;231(4):563-9.

30. Gray KN, Raulston GL, Gleiser CA, et al. Histologic classification as an indication of therapeutic response in malignant lymphoma of dogs. J Am Vet Med Assoc 1984;184(7):814-7.

31. Van Vechten M, Helfand SC, Jeglum KA. Treatment of relapsed canine lymphoma with doxorubicin and dacarbazine. J Vet Intern Med 1990;4(4):187-91.

32. Zucali PA, Bertuzzi A, Parra HJ, et al. The "old drug" dacarbazine as a second/third line chemotherapy in advanced soft tissue sarcomas. Invest New Drugs 2008;26(2):175-81.

33. Pearl ML, Inagami M, McCauley DL, et al. Mesna, doxorubicin, ifosfamide, and dacarbazine (MAID) chemotherapy for gynecological sarcomas. Int J Gynecol Cancer 2002;12(6):745-8.

34. Elias AD, Antman KH. Doxorubicin, ifosfamide, and dacarbazine (AID) with mesna uroprotection for advanced untreated sarcoma: a phase I study. Cancer Treat Rep 1986;70(7):827-33.

35. Etcubanas E, Horowitz M, Vogel R. Combination of dacarbazine and doxorubicin in the treatment of childhood rhabdomyosarcoma. Cancer Treat Rep 1985;69(9):999-1000.

36. Choi TK, Ng A, Wong J. Doxorubicin, dacarbazine, vincristine, and cyclophosphamide in the treatment of advanced gastrointestinal leiomyosarcoma. Cancer Treat Rep 1985;69(4):443-4.

37. Hahn KA. Vincristine sulfate as single-agent chemotherapy in a dog and a cat with malignant neoplasms. J Am Vet Med Assoc 1990; 197(4):504-6.

38. Gagner JP, Yim JH, Yang GC. Fine-needle aspiration cytology of epithelioid angiosarcoma: a diagnostic dilemma. Diagn Cytopathol 2005;33(6):429-33.

39. Delacruz V, Jorda M, Gomez-Fernandez C, et al. Fine-needle aspiration diagnosis of angiosarcoma of the spleen: a case report and review of the literature. Arch Pathol Lab Med 2005;129(8):1054-6.

40. Nguyen GK, Husain M. Fine-needle aspiration biopsy cytology of angiosarcoma. Diagn Cytopathol 2000;23(2):143-5.

41. Noda T, Watanabe T, Kohda A, et al. Chronic effects of a novel synthetic anthracycline derivative (SM-5887) on normal heart and doxorubicin-induced cardiomyopathy in beagle dogs. Invest New Drugs 1998;16(2):121-8.

42. Danesi R, Del Tacca M, Bernardini N, et al. Evaluation of the JT and corrected JT intervals as a new ECG method for monitoring doxorubicin cardiotoxicity in the dog. J Pharmacol Methods 1989; 21(4):317-27.

43. Veterinary Co-operative Oncology Group (VCOG). Veterinary Co-operative Oncology Group - Common Terminology Criteria for Adverse Events (VCOG-CTCAE) following chemotherapy or biological antineoplastic therapy in dogs and cats v1.0. Vet Comp Oncol 2004;2(4):195-213.

44. Simon R. Designs for efficient clinical trials. Oncology (Williston Park) 1989;3(7):43-9, 51-3. 
45. Simon R. Optimal two-stage designs for phase II clinical trials. Control Clin Trials 1989;10(1):1-10.

46. Long L, Dolan ME. Role of cytochrome P450 isoenzymes in metabolism of $\mathrm{O}(6)$-benzylguanine: implications for dacarbazine activation. Clin Cancer Res 2001;7(12):4239-44.

47. Trepanier LA. Cytochrome P450 and its role in veterinary drug interactions. Vet Clin North Am Small Anim Pract 2006;36(5): 975-85, v.

48. Tenmizu D, Noguchi K, Kamimura H. Elucidation of the effects of the CYP1A2 deficiency polymorphism in the metabolism of 4-cyclohexyl-1-ethyl-7-methylpyrido[2,3-d]pyrimidine-2-(1h)-one (YM-64227), a phosphodiesterase type 4 inhibitor, and its metabolites in dogs. Drug Metab Dispos 2006;34(11):1811-6.
49. Kamimura H. Genetic polymorphism of cytochrome P450s in beagles: possible influence of CYP1A2 deficiency on toxicological evaluations. Arch Toxicol 2006;80(11):732-8.

50. Tenmizu D, Endo Y, Noguchi K, et al. Identification of the novel canine CYP1A2 $1117 \mathrm{C}>\mathrm{T}$ SNP causing protein deletion. Xenobiotica 2004;34(9):835-46.

51. Mise M, Hashizume T, Matsumoto S, et al. Identification of nonfunctional allelic variant of CYP1A2 in dogs. Pharmacogenetics 2004; 14(11):769-73.

52. Mise M, Yadera S, Matsuda M, et al. Polymorphic expression of CYP1A2 leading to interindividual variability in metabolism of a novel benzodiazepine receptor partial inverse agonist in dogs. Drug Metab Dispos 2004;32(2):240-5. 